\title{
A genome-wide association study identifies candidate loci associated to syringomyelia secondary to Chiari-like malformation in Cavalier King Charles Spaniels
}

Frédéric Ancot ${ }^{1 \dagger}$, Philippe Lemay ${ }^{1+}$, Susan P. Knowler ${ }^{2}$, Karen Kennedy ${ }^{3}$, Sandra Griffiths ${ }^{4}$, Giunio Bruto Cherubini ${ }^{5}$, Jane Sykes ${ }^{6}$, Paul J. J. Mandigers ${ }^{7}$, Guy A. Rouleau ${ }^{8}$, Clare Rusbridge ${ }^{2,9}$ and Zoha Kibar ${ }^{1 *}$ (D)

\begin{abstract}
Background: Syringomyelia (SM) is a common condition affecting brachycephalic toy breed dogs and is characterized by the development of fluid-filled cavities within the spinal cord. It is often concurrent with a complex developmental malformation of the skull and craniocervical vertebrae called Chiari-like malformation (CM) characterized by a conformational change and overcrowding of the brain and cervical spinal cord particularly at the craniocervical junction. CM and SM have a polygenic mode of inheritance with variable penetrance.

Results: We identified six cranial T1-weighted sagittal MRI measurements that were associated to maximum transverse diameter of the syrinx cavity. Increased syrinx transverse diameter has been correlated previously with increased likelihood of behavioral signs of pain. We next conducted a whole genome association study of these traits in 65 Cavalier King Charles Spaniel (CKCS) dogs (33 controls, 32 with extreme phenotypes). Two loci on CFA22 and CFA26 were found to be significantly associated to two traits associated with a reduced volume and altered orientation of the caudal cranial fossa. Their reconstructed haplotypes defined two associated regions that harbor only two genes: PCDH17 on CFA22 and ZWINT on CFA26. PCDH17 codes for a cell adhesion molecule expressed specifically in the brain and spinal cord. ZWINT plays a role in chromosome segregation and its expression is increased with the onset of neuropathic pain. Targeted genomic sequencing of these regions identified respectively 37 and 339 SNPs with significantly associated $P$ values. Genotyping of tagSNPs selected from these 2 candidate loci in an extended cohort of 461 CKCS (187 unaffected, 274 SM affected) identified 2 SNPs on CFA22 that were significantly associated to SM strengthening the candidacy of this locus in SM development.
\end{abstract}

Conclusions: We identified 2 loci on CFA22 and CFA26 that contained only 2 genes, PCDH17 and ZWINT, significantly associated to two traits associated with syrinx transverse diameter. The locus on CFA22 was significantly associated to SM secondary to CM in the CKCS dog breed strengthening its candidacy for this disease. This study will provide an entry point for identification of the genetic factors predisposing to this condition and its underlying pathogenic mechanisms.

Keywords: Syringomyelia, Chiari malformation, CKCS dog breed, Cranial MRI measurements, Whole genome association study

\footnotetext{
* Correspondence: zoha.kibar@recherche-ste-justine.qc.ca

${ }^{\dagger}$ Equal contributors

${ }^{1}$ Department of Neurosciences, CHU Sainte Justine Research Center,

University of Montréal, 3175 Cote-Sainte-Catherine, Room 3.17.006, Montreal,

QC H3T 1C5, Canada

Full list of author information is available at the end of the article
}

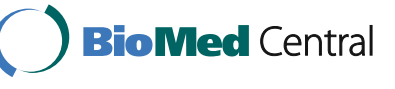

(c) The Author(s). 2018 Open Access This article is distributed under the terms of the Creative Commons Attribution 4.0 International License (http://creativecommons.org/licenses/by/4.0/), which permits unrestricted use, distribution, and reproduction in any medium, provided you give appropriate credit to the original author(s) and the source, provide a link to the Creative Commons license, and indicate if changes were made. The Creative Commons Public Domain Dedication waiver (http://creativecommons.org/publicdomain/zero/1.0/) applies to the data made available in this article, unless otherwise stated. 


\section{Background}

Canine syringomyelia (SM) is a painful condition where fluid-containing cavities (syrinx or syringes) develop within the parenchyma of the spinal cord and which progress over time $[1,2]$. Depending on the site of spinal cord damage, SM may result in behavioral signs of pain, fictive scratching, scoliosis, weakness and sensory deficits [3]. Approximatively 70\% of older Cavalier King Charles spaniels (CKCS) have MRI signs of SM. This high percentage seems to correlate with another condition present ubiquitously in this breed called Chiari-like malformation (CM) [2]. CM is a complex developmental malformation of the skull and cranial cervical vertebrae that is characterized by rostro-caudal bony insufficiency resulting in conformational changes and overcrowding of the brain and cervical spinal cord particularly at the craniocervical junction. Obstruction of the foramen magnum and cerebrospinal fluid (CSF) channels is hypothesized to be pivotal in the pathogenesis of SM [4-7].

In the CKCS, risk of SM has been shown to be associated with increased brachycephaly with rostrocaudal doming i.e. a heightened cranium that slopes caudally [8] and reduced skull base due to craniosynostosis or premature skull suture closure [9]. The underlying pathogenic mechanisms proposed for the development and progression of SM secondary to CM remain poorly understood and even controversial complicating the interpretation of many clinical observations and the selection of the appropriate treatment protocols. Current prevailing hydrodynamic theories generally assume that syrinx fluid is CSF that has entered the cord as a result of perturbations of pulsations in the subarachnoid space caused by overcrowding of neural parenchyma in the caudal part of the cranial fossa and the cervical vertebral canal as seen in CM [10].

Studies on the inheritance of SM have shown that it is a complex trait with a moderately high heritability [11]. The genetic origin of $\mathrm{CM}$ and its role in $\mathrm{SM}$ remain to be identified. Incomplete penetrance and variability of clinical signs in both $\mathrm{CM}$ and SM seem to indicate a polygenic mode of inheritance $[11,12]$. The genetic approach widely used to investigate complex disorders is a genome-wide association study (GWAS) that aims at identifying genes or SNPs that determine the disease even though each gene contributes only a small fraction to the disease process. This strategy was applied successfully in dogs and permitted the identification of loci associated with osteoarthritis of hip joints [13], hip and elbow dysplasia [14], and related BMP3 (BONE MORPHOGENETIC PROTEIN 3) variations to skull diversity [15]. Particularly, an association study was successfully used to identify loci associated with $\mathrm{CM}$ in the Griffon Bruxellois (GB) breed. A total of 14 quantitative skull and atlas measurements were taken and were tested for association to $\mathrm{CM}$ in the $\mathrm{GB}$. Significant associations were identified between specific traits and $\mathrm{CM} / \mathrm{SM}$ status in $\mathrm{GB}[16,17]$, a mixed GB breed [18] and CKCS cohorts [6]. A GWAS in the GB cohort identified one locus on CFA2 (CFA, Canis Familiaris autosome) strongly associated to the height of the cranial fossa and another locus on CFA14 associated to both the height of the caudal cranial fossa (reduced supraoccipital bone) and brachycephaly [17]. These two loci were significantly associated to $\mathrm{CM}$ further strengthening their candidacy. In this study, we conducted a GWAS to identify genomic regions that predispose to SM secondary to CM in the CKCS dog breed.

\section{Methods}

\section{Cohort and phenotypic traits}

A cohort of $96 \mathrm{CM}$ affected CKCS with DNA consisting of 40 males (26 affected and 14 unaffected) and 56 females (35 affected and 21 unaffected) with an average age of 5.5 \pm 2.5 years was included in the quantitative study investigating CM (Additional file 1: Table S1). This cohort was part of a larger CKCS cohort used by the same group to characterize painful $\mathrm{CM}$ and secondary SM for which DNA was available [6]. DICOM (Digital Imaging and Communications in Medicine) T1-weighted midsagittal MRI of the brain and cervical region of these 96 dogs allocated with an ID were analyzed. The minimum inclusion criterion was visualization of the hindbrain to the level of the interthalamic adhesion to the cervical vertebrae $4 / 5$ intervertebral disc space. Only DICOM images accompanied by details of birth, date of MRI and identity microchip number were used. The MRI studies were loaded into DICOM viewing software eFILM workstation (Merge Healthcare 900 Walnut Ridge Drive, Hartland, WI 53029 USA). CM/SM status was determined by author CR by noting the presence of CM and SM on sagittal T1 and T2W weighted images and then, if SM was present, determining the maximum internal syrinx transverse diameter (STD) from transverse T1 weighted images of the cervical spinal cord. The smallest unit of measurement in eFILM is $1 \mathrm{~mm}(\mathrm{~mm})$. These criteria are in accordance with the British Veterinary Association/ Kennel Club guidelines for screening for SM (https://www.bva.co.uk/Canine-HealthSchemes/CM-SM-Scheme/). SM severity was established according to the STD as wide STD has been previously associated with clinical signs of pain, fictive scratch and scoliosis [19]. The 96 CKCSs were separated in 3 categories that took account of late onset condition of SM: normal (35 dogs without syrinx or central canal dilation (STD $=0 \mathrm{~mm}$ over 5 years of age), intermediate (29 dogs with STD $=1$ or $2 \mathrm{~mm}$ over 5 years of age) or severe (32 dogs with STD $\geq 3 \mathrm{~mm}$ any age). 
Next, using a DICOM reading software (Mimics ${ }^{\circ} 14.12$, Materialise, Belgium), a total of 11 structures were defined and 27 lines, angles and ratios were measured by SPK, initially blinded to SM status (Fig. 1, Additional file 1: Table S1). The mapping of the hindbrain and craniocervical junction was adapted from previous genetic and conformational studies undertaken in the Griffon Bruxellois [16, 17]. Dogs with narrow syringes or small central canal dilation are more likely to be asymptomatic at least with regard to the syrinx. The CKCS breed is very variable in size and head-shape. In view of this, two ratios were taken which reflected the size and shape of the caudal fossa and relating this to the height of the cranial fossa (f-diameter). The first ratio was the $\mathrm{f}$-diameter $(\mathrm{F}-\mathrm{d})$ divided by the distance across the foramen magnum from the caudal point of the basioccipital to the rostral point of the atlas (line $\mathrm{BC}$ ) and the second was F-d divided by the height of the supraoccipital bone (line CD) (Fig. 1). Since the MRI of the forebrain and olfactory bulb was not always available for analysis, in order to take account of the impact of brachycephaly and compensatory rearrangement of parenchyma and reduced caudal fossa, the two angles which most reflected these deviations in the hindbrain, angle 4 and angle 7 were combined $(L 4+L 7)$ (Fig. 2). The hypothesis being the smaller these two angles were, the greater the deviation and reduction in the neural parenchyma of the hindbrain and craniocervical junction.

\section{Genotyping across the genome}

A total of 96 CKCSs were genotyped with CanineHD Genotyping Beadchip (Illumina Inc., San Diego, CA, USA) which contained 173,662 SNPs. These SNPs were analysed using genome studio and filtered for SNPs with a minor allele frequency $>0.05$, a SNP genotyping rate of 0.9 and an individual genotyping rate of 0.9 using Plink V1.07. A total of 85,647 SNPs were excluded for lack of informativeness in the CKCS breed $(\mathrm{MAF}<0.05)$. Three dogs had a genotyping rate under 0.9 and were therefore excluded from further analysis resulting in 93 dogs (Initial cohort: 39 affected, 54 unaffected, 39 males, 54 females). To remove potential bias associated with ambiguous phenotypes, dogs with STD less than $3 \mathrm{~mm}$ were removed from the analysis. This resulted in a final cohort of 65 dogs (32 affected, 33 unaffected, 26 males, 39 females) genotyped with 88,015 SNPs.

\section{Targeted next generation sequencing}

The 2 loci of $0.17 \mathrm{Mb}$ and $0.8 \mathrm{Mb}$ on CFA22 and CFA26 associated to ratio F-diameter (F-d)/BC and $L 4+L 7$ respectively were sequenced in the same 65 dogs used for GWAS using SeqCap Custom relaxed probe set library preparation specifically designed by Roche Nimblegen (Madison, WI, USA) and subsequently subjected to 100 base pair, paired end sequencing on the Illumina HiSeq2000 Platform at the McGill University and Génome Québec Innovation Centre. Using an SSAHA algorithm, the baits contained 3313 probes with up to 20 close

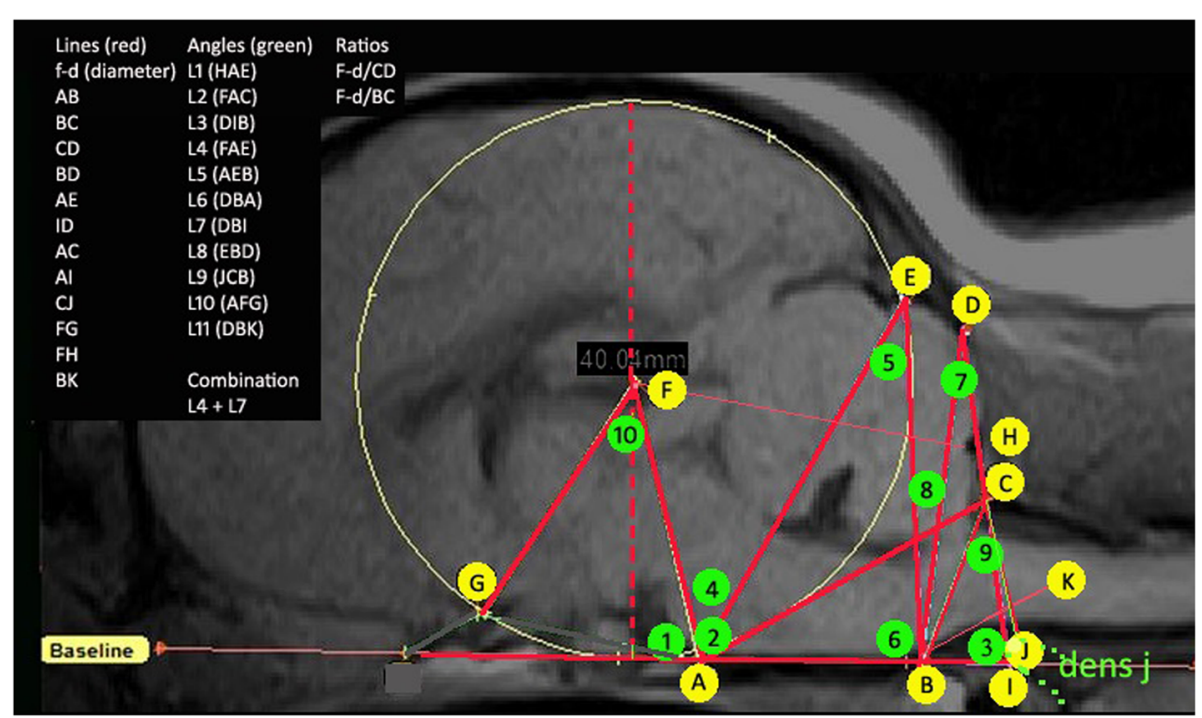

Fig. 1 Morphometric measurements of a Cavalier King Charles Spaniels whole brain. Measurements were chosen to best reflect the possible morphological changes observed in SM. All measurements start from one of these points: a dorsum of sphenoid-occipital synchondrosis, $\mathbf{b}$ basion of basioccipital bone, $\mathbf{c}$ rostral edge of the dorsal lamina of the atlas, $\mathbf{d}$ junction between supraoccipital bone and occipital crest, e most dorsal point of intersection of the cerebellum with the occipital lobe circle, $\mathbf{f}$ center of occipital lobe circle, $\mathbf{g}$ point at which the optic nerve deviates into the optic canal, $\mathbf{h}$ rostral edge of supra-occipital bone, $\mathbf{i}$ intersection point with the extended AB baseline caudally with extended line DC ventrally, $\mathbf{j}$ most rostral aspect of the dens of the axis bone and $\mathbf{k}$ extended line from point $\mathrm{B}$ along the best fit line of the ventral medulla oblongata to where it changes angle to the spinal cord 


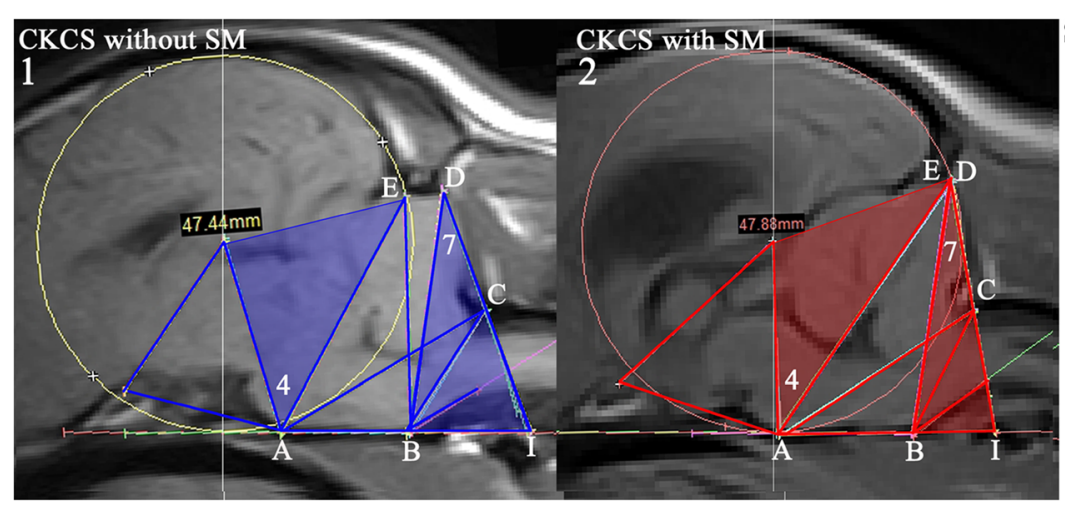

Superimposed morphometrics of dogs 1 and 2

Fig. 2 Cavalier King Charles Spaniel with and without SM illustrating differences in the size and arrangement of a combination of angles 4 and 7 $(L 4+L 7)$. Angles 4 and 7 are smaller in the CKCS with SM (red) as compared to CKCS without SM (blue) reflecting a reduced cranial caudal fossa and rearrangement of neural parenchyma. Right panel, a schematic Fig. of the occipital circle with centre F (grey) and angles $4+7$ of the CKCS with SM (red) that have been superimposed on the CKCS without SM (blue). A, dorsum of sphenoid- occipital synchondrosis; B, basion of basioccipital bone; D, junction between supraoccipital bone and occipital crest; E, most dorsal point of intersection of the cerebellum with the occipital lobe circle; F, center of occipital lobe circle

matches in the genome for the purposes of providing maximum coverage. The vast majority of the probes were unique, with a few probes that had a greater degree of multi-locus homology to increase coverage in all regions. Reads were mapped to the genome (CanFam3.1 release September 2011) with Burrows-Wheeler Aligner (BWA). Duplicated reads were removed, the rest were locally realigned using GATK v2.6.4 and annotated using Annovar. The average read depth for the targeted regions was $151,01 \mathrm{X}(89,32 \mathrm{X}-208,32 \mathrm{X})$ with $99,58 \%$ of targeted regions covered at greater than $20 \mathrm{X}$.

\section{Genotyping with tagSNPs}

Tagging SNPs were selected to maximize coverage of each locus. They were identified using the tagger routine in the Haploview V4.2 software with a maximum $r^{2}$ of 0.2. Two tagSNPs were amplified on CFA22 at position $13,804,718$ and 13,933,606 (rs23040347) with the probes GGATTACAGAAGTCACAGTCGAAAGACTGGGAAAG AGACACCAGAGCTCCAAGTTTATAAAGTTGTATTTT AAAGATTCAGTGATGTCTGAGGAATGAAATGGGAT GAGGAAGGAAAAATTATGCACTAGGAGCAATGTTT TCTGTCTTC[T/A]GGAATGGGAAGTGAGATGAACAG CATAGGAGATTTGAAAGGTAGCAAACAATCCAGGA GACCTACAGGCCCGTGATCAATGACTTATAAGATGA TATTAAGGAAACATTATATATGATACTATATCCTCCT TTGAGAGTCTGTATGCATT and TTAATCTTCAAAAC GGCCCAATGAGGATATCCCCATTTTGCAGATGAAG AATGAAGGAACGTTGAAGTTCAGTGATTTGATCCA GAATAAGTGCAGTGAGACTTCAGATCCAGCTATGG GGTTTGCCAAATTCAATCTCTGCCCTTCCTC[A/C]C TATTCTTAACCGCCAAATATTATTTATATTTGTAAGA ATGCAGTTTTAAAGGTTGAAATTTTCAACTTCTCAC ACAGAGCAGATAGCTGGAGACGAAGATGGTAGGAC TGCTCTCTCATTGCCTGCATTGTGCTCTCTGAGTAG
TGAAA respectively. Two SNPs were also amplified on CFA26 at position 32,757,080 (rs23302138) and 32,797,595 with the probes TGTCCTCCTGGCTTCTGAGGGGG TGGGTGCGGGGCCTGGAGGCCCAGAGGGGAACAG GATGTGGCCACAGGATGGAGAGCTGACTTGTGCAC AGGGGCCTGTGTGGGTCAGTCTGTGTCCCCGGCAC CCCTGAAGCTGCAGGTGTCTCAG[T/C]AGAGCCCC TCAGTGGGTAACTCTGCCCCCAATTCCCTCCTTGGA GACTGCATCTCCTCCTGTGCCTCCTGCAAGTCGCT GTCAGCTTCCCTCCCCTGAGGTCTGACGCCTCCTG CAGGAAGTTCTCTGGGATTGGATCTCAAAATGGTG $\mathrm{C}$ and CATGAGTTGGAAGGGCAGTTAAGGGCAGAAG GACTTAGAGGCGGAGAGCATAGAGAAGGAAAAGG CACGATGGTGTGTTTGATTATCTCCCCCTCTCCATT CTCATGGTGCCACCTATCCTAATTCCAGTTCGTATT ATCATAGGTCTCA[C/T]TCCACCAATAACGTCTCAA TCACACACACCATGTCCTGTCTTCTCGTTGGTCTGT GCTATGATCTGTGTGGTTCTTCTCTTTCCCAGGAG GCCAGATCTGTATTTTGCTGATTACAATCTACTCTT TAATTCTGGATTGAATTGCTAACT respectively. Genotyping was performed using the TaqMan assay (Applied Biosystems) on 393 CKCS dogs (187 SM unaffected, 274 SM affected).

\section{Statistical analyses}

Initial GWAS analysis was done in 2 phases: association of the quantitative traits (skull and cranial cervical measurements) to disease, followed by association of SNPs to these quantitative traits. Association of the quantitative traits to the disease was done using a linear regression with a type III sum of squares in R V3.0.1 [20] and age as a covariate. Due to the strong association of STD size and age, inclusion of age as a covariate was used to correct for its potential confounding effect. Association of SNPs to the quantitative traits was done using a 
mixed linear model including age as a covariate and potential stratification as a random effect which was corrected using a genomic relationship matrix using GEMMA V0.94 [21]. All $P$ values obtained from the association were corrected together for multiple testing using a storey's q value method [22]. Haplotypes surrounding these SNPs were reconstructed using Haploview V4.2 [23] and associations with the initial quantitative trait were run using Plink V1.07 linear routine [24] with age as a covariate. Correction was applied using 10,000 permutations. Plink V1.07 logistic regression routine using age as a covariate was used to test for association between tag SNPs and SM and Bonferroni was used to correct for multiple testing $(0.05 / 4=$ 0.0125).

\section{Results}

\section{Association of skull measurements to SM in the CKCS} breed

The complex skull morphology of the CKCS breed was investigated using 27 lines, angles and ratios as well as age, gender and ventricular dilatation on a cohort of 96 dogs (Figs. 1 and 2, Additional file 1: Table S1). Level of affliction of dogs was defined by the STD size. A linear regression including age as a covariate was used to associate traits and STD size. STD size did not show any association to gender (Table 1). A total of 6 measurements consisting of line AE, line AI, angle 3, angle 7, ratio F-d/ $\mathrm{BC}$ and $L 4+L 7$ all showed a significant association to STD size $\left(\mathrm{P}_{\text {bonferroni }}<0.0019\right)$ and were therefore further investigated (Fig. 3 and Table 1).

\section{Genome-wide association study of SM in CKCS}

A GWAS using a mixed linear model with age as a covariate was applied on the previously identified traits (Line AE, line $\mathrm{AI}$, angle 3 , angle 7 , ratio $\mathrm{F}-\mathrm{d} / \mathrm{BC}$ and $L 4$ + L7). This resulted in the identification of a group of 13 SNPs on CFA15 (BICF2S23761321, BICF2G630435380, BICF2S22961368, BICF2G630437186, BICF2G6304371 78, BICF2G630437135, BICF2G630437112, BICF2G6304 37075, BICF2G630437073, BICF2S23311892, BICF2G63 0437043, BICF2G630437038, BICF2G630437002) associated to ratio F-d/BC under a FDR of 0.05 (all $P=0.03754$ ) and two SNPs on CFA26 (BICF2P174010, BICF2P152116), which were significantly associated to $L 4+L 7$ under a FDR of 0.05 (both $P=0.03754$ ) (Fig. 4 and Table 2).

Multiple SNPs were suggestive of association with FDR corrected scores between 0.05 and 0.1 (Additional file 2: Table S2). By comparing the list of these "borderline" SNPs to a previous association study of CM in the GB breed conducted by our group [17], we were able to identify an overlap with only one SNP on CFA22, BICF2P1045632, that was associated with ratio F-d/BC with a $P$ value after FDR of 0.07846 in the present study.
Table 1 Quantitative traits that are significantly associated $(P$ value $<0.05)$ to syrinx transverse diameter following linear regression and multiple testing correction

\begin{tabular}{|c|c|c|}
\hline Trait & Raw $P$ value & Bonferroni corrected $P$ value* \\
\hline Gender & 0.680431 & 1 \\
\hline Age & 8.01E-06 & 0.000216 \\
\hline F-Diameter & 0.005901 & 0.159332 \\
\hline Line $A B$ & 0.609389 & 1 \\
\hline Line $B C$ & 0.172273 & 1 \\
\hline Line CD & 0.815288 & 1 \\
\hline Line BD & 0.068142 & 1 \\
\hline Line AE & 0.00124 & 0.033479 \\
\hline Line ID & 0.17867 & 1 \\
\hline Line $A C$ & 0.084552 & 1 \\
\hline Line Ai & $7.82 \mathrm{E}-05$ & 0.002113 \\
\hline Line CJ & 0.014438 & 0.389822 \\
\hline Line $\mathrm{FH}$ & 0.952342 & 1 \\
\hline Line FG & 0.151877 & 1 \\
\hline Line BK & 0.03617 & 0.976588 \\
\hline Angle L1 (hae) & 0.769033 & 1 \\
\hline Angle L2 (fac) & 0.038195 & 1 \\
\hline Angle L3 (dib) & $8.11 \mathrm{E}-06$ & 0.000219 \\
\hline Angle L4 (fae) & 0.124912 & 1 \\
\hline Angle L5 (aeb) & 0.329471 & 1 \\
\hline Angle $L 6$ (dba) & 0.278602 & 1 \\
\hline Angle L7 (bdi) & $1.01 \mathrm{E}-05$ & 0.000272 \\
\hline Angle L8 (ebd) & 0.861691 & 1 \\
\hline Angle L9 (jcb) & 0.136343 & 1 \\
\hline Angle L10 (afg) & 0.036817 & 0.994072 \\
\hline Angle L11 (ebk) & 0.100105 & 1 \\
\hline Ratio F-d/CD & 0.473593 & 1 \\
\hline Ratio F-d/BC & 0.00172 & 0.046441 \\
\hline$L 4+L 7$ & 1.33E-05 & 0.000358 \\
\hline
\end{tabular}

${ }^{*} P$ values in bold represent significant association

This SNP maps to a region that was identified as suggestive of association with the line $\mathrm{BC}$ in the previously studied GB breed [17]. A replication between two unrelated cohorts from two different breeds offers strong evidence for association strengthening the candidacy of this SNP and suggesting the need for inclusion in the following genetic studies. The other SNPs that did not reach statistical significance in this study and that were not replicated in our previous association study of CM in GB dogs require further genetic analyses in larger cohorts.

\section{Haplotype analysis of the candidate SM loci}

Using Haploview V4.2, blocks of linkage disequilibrium (LD) surrounding the SNPs significantly associated to 

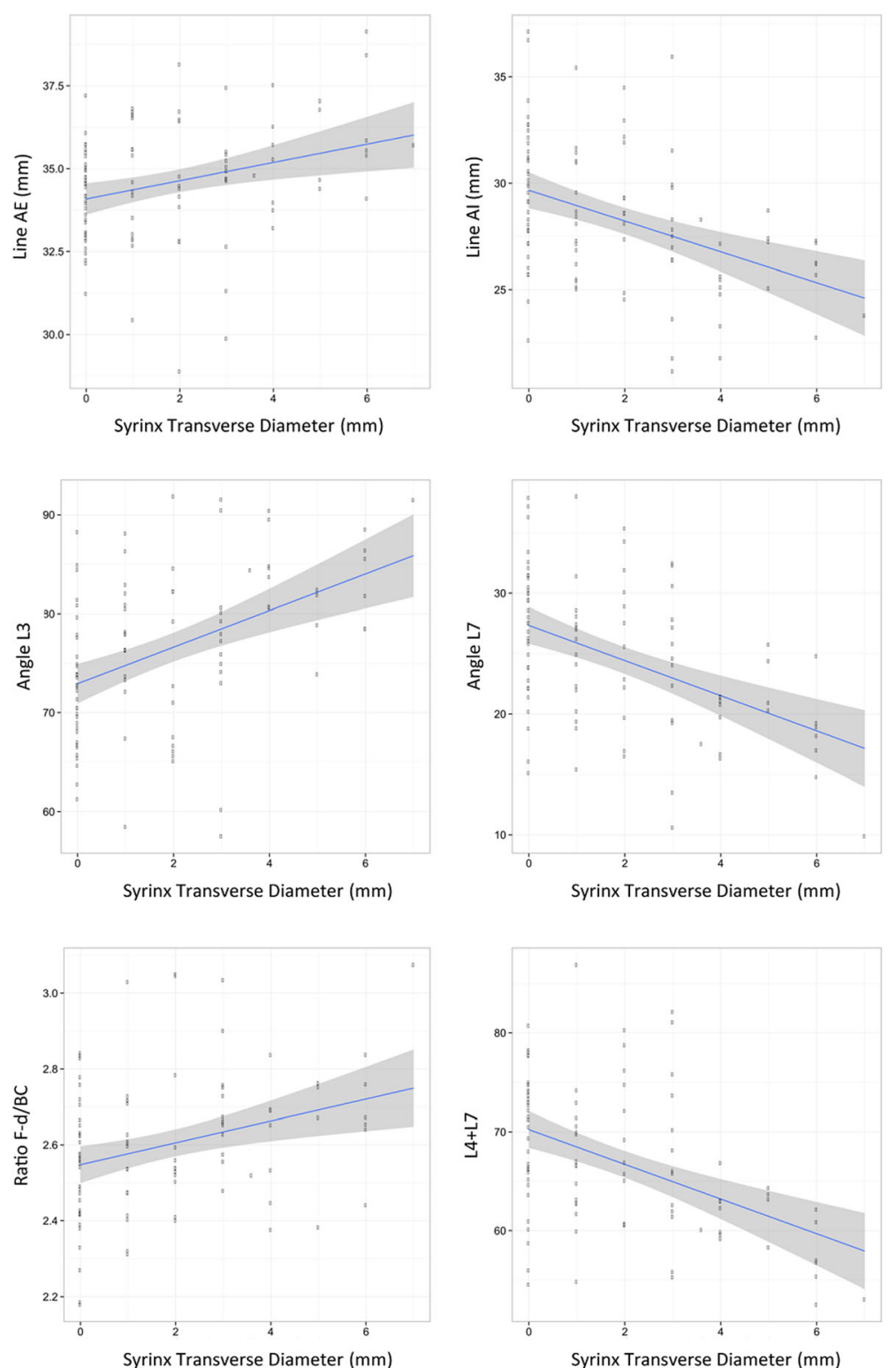

Fig. 3 Linear regression of traits and STD size including age as a covariate. A total of 6 measurements consisting of line AE, line Al, angle 3, angle 7, ratio $F-d / B C$ and $L 4+L 7$ all showed a significant association to STD size $\left(P_{\text {bonferroni }}<0.0019\right)$

$L 4+L 7$ and ratio $\mathrm{F}-\mathrm{d} / \mathrm{BC}$ were defined. A first $\mathrm{LD}$ block of $1.7 \mathrm{Mb}$ surrounding SNPs associated to ratio F-d/ BC was identified on CFA15 from $24,537,882$ bp to 26,252,411 bp (Additional file 3: Fig. S1). A second LD block of $0.8 \mathrm{Mb}$ surrounding SNPs associated to $L 4+L 7$ was identified on CFA26 from position $32,226,403$ bp to $33,034,398$ bp. A last LD block of $0.17 \mathrm{Mb}$ surrounding the SNP associated with ratio $\mathrm{F}-\mathrm{d} / \mathrm{BC}$ and identified previously in the GB cohort was identified on CFA22 from $14,107,661$ bp to $14,276,181 \mathrm{bp}$. The three haplotypes were analysed using linear regression that included age as a covariate and looked at the potential association of these blocks with their respective traits. Linear regressions are known to be sensitive to rare haplotypes associated to extreme measurements; therefore, rare haplotypes with a frequency under 0.05 were excluded. This resulted in the identification of three haplotypes, one on CFA22 $(P=0.009599)$ and two on CFA26 $(P=0.01067$ and 0.00231$)$, that were significantly associated to ratio $\mathrm{F}-\mathrm{d} / \mathrm{BC}$ (Table 3 ) and $L 4+L 7$ (Table 4) respectively (Fig. 5). The strong association of these haplotypes and SNPs with their respective traits combined with the strong association of these traits with the STD size support the implication of these regions in the development of SM. No significantly associated haplotypes were identified on CFA15 excluding his region from further analyses. 


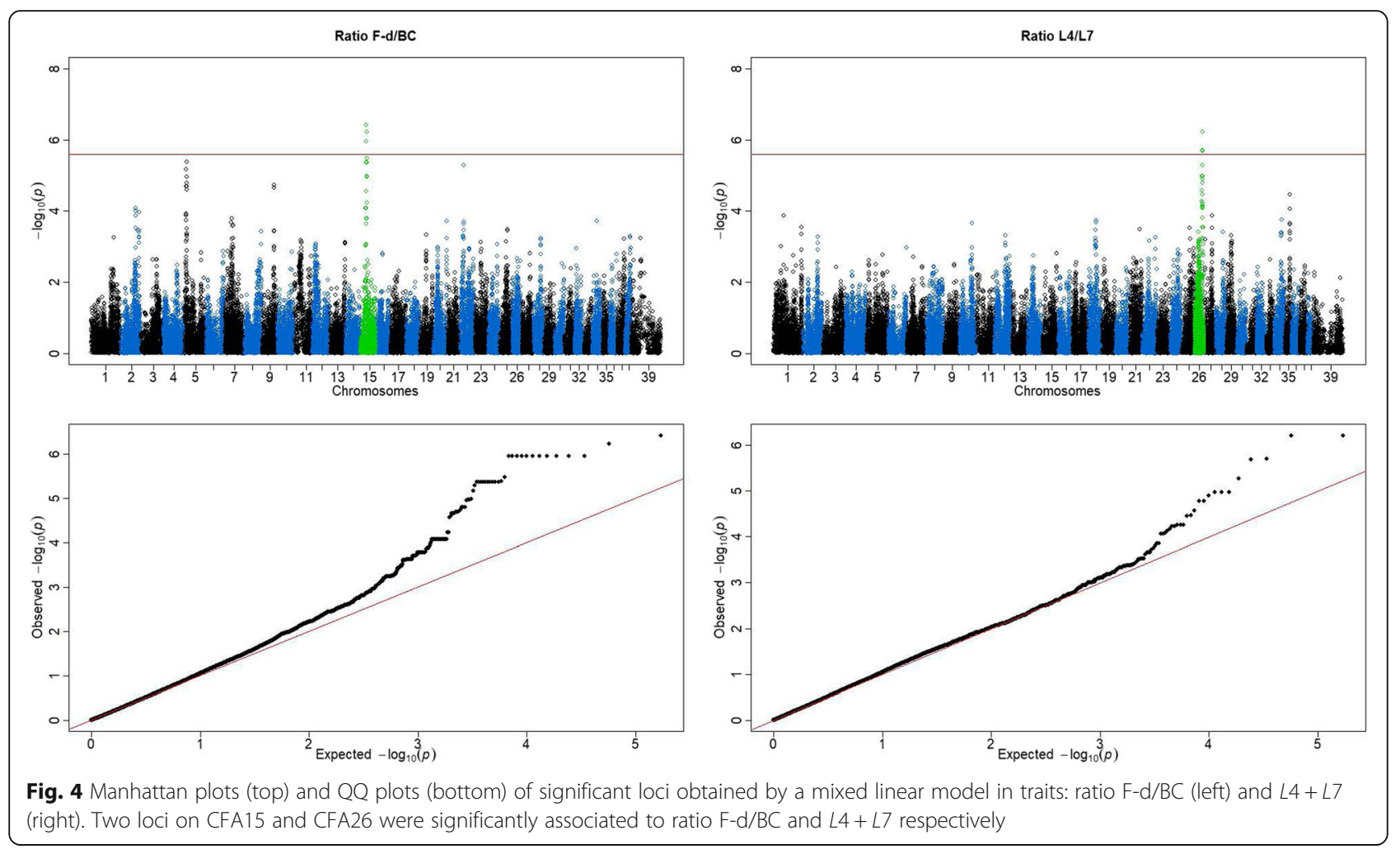

\section{Targeted next generation sequencing of the SM-}

\section{associated loci on CFA22 and CFA26}

Each of the SM- associated regions harbored only one coding gene: PCDH17 (PROTO-CADHERIN 17) on CFA22 and ZWINT (ZW1O INTERACTING KINETOCHORE PROTEIN) on CFA26. In order to identify potential SM -predisposing mutations, both CFA22 and
CFA26 candidate regions were submitted to targeted next generation sequencing. The reads of the sequences obtained from the 65 CKCS were aligned on CanFam 3.1 resulting in 5608 SNPs on CFA22 and 10,814 SNPs on CFA26. Except for one SNP (rs2305483), that was identified in the coding region of $P C D H 17$ and that was non-conserved and synonymous, all identified SNPs

Table 2 Loci significantly associated to ratio F-d/BC and L4+L7 in the mixed linear model

\begin{tabular}{|c|c|c|c|c|}
\hline Chr & SNP & Position & Raw $P$ value & FDR corrected $P$ value \\
\hline 15 & BICF2S23761321 & $28,798,671$ & $3.81 \mathrm{E}-07$ & 0.037545 \\
\hline 15 & BICF2G630435380 & $29,147,043$ & 5.87E-07 & 0.037545 \\
\hline 15 & BICF2S22961368 & $26,586,223$ & 1.11E-06 & 0.037545 \\
\hline 15 & BICF2G630437186 & $26,599,059$ & $1.11 \mathrm{E}-06$ & 0.037545 \\
\hline 15 & BICF2G630437178 & $26,605,637$ & 1.11E-06 & 0.037545 \\
\hline 15 & BICF2G630437135 & $26,619,845$ & $1.11 \mathrm{E}-06$ & 0.037545 \\
\hline 15 & BICF2G630437112 & $26,623,178$ & 1.11E-06 & 0.037545 \\
\hline 15 & BICF2G630437075 & $26,645,302$ & $1.11 \mathrm{E}-06$ & 0.037545 \\
\hline 15 & BICF2G630437073 & $26,645,969$ & 1.11E-06 & 0.037545 \\
\hline 15 & BICF2S23311892 & $26,690,382$ & $1.11 \mathrm{E}-06$ & 0.037545 \\
\hline 15 & BICF2G630437043 & $26,734,763$ & 1.11E-06 & 0.037545 \\
\hline 15 & BICF2G630437038 & $26,738,248$ & $1.11 \mathrm{E}-06$ & 0.037545 \\
\hline 15 & BICF2G630437002 & $26,797,343$ & $1.11 \mathrm{E}-06$ & 0.037545 \\
\hline 26 & BICF2P174010 & $32,735,128$ & 5.99E-07 & 0.037545 \\
\hline 26 & BICF2P152116 & $32,738,238$ & 5.99E-07 & 0.037545 \\
\hline
\end{tabular}


Table 3 Raw and permutation P values of the F-d/BC-associated haplotypes in the CFA22 candidate region at 14107661-14276181 bp

\begin{tabular}{lllll}
\hline Name & Haplotype & Frequency & Raw $P$ value & Corrected $P$ value \\
\hline $\mathbf{1}$ & AGCCGTCCCTTG & 0.431 & 0.956 & 1 \\
$\mathbf{2}$ & GAACATTGCGTA & 0.351 & 0.262 & 0.7261 \\
$\mathbf{3}$ & AGCTGGCCTTCG & 0.100 & 0.00153 & 0.009599 \\
$\mathbf{4}$ & GGACAGCCTTCG & 0.041 & 0.0644 & 0.2626 \\
$\mathbf{5}$ & GAACATTGCTTA & 0.062 & 0.223 & 0.659 \\
\hline
\end{tabular}

resided in intergenic and deep intronic regions. Using improved coverage of the region, linkage disequilibrium blocks were reevaluated based on the significant SNPs in the regions. A total of 37 and 339 SNPs were defined as significantly associated to $\mathrm{F}-\mathrm{d} / \mathrm{BC}$ and $L 4+L 7$ respectively. Based on the hypothesis that causative SNPs would be significantly associated to their respective trait, these SNPs refined the regions of interest to $13,785,828$ $14,183,295$ bp (397,467 bp) on CFA22 and 32,721,057 bp to $33,094,292 \mathrm{bp}(373,235 \mathrm{bp})$ on CFA26.

\section{Genotyping of an extended CKCS cohort with SNPs significantly associated to ratio $\mathrm{F}-\mathrm{d} / \mathrm{BC}$ and $L 4+L 7$} To investigate the potential association between the identified SNPs and SM, a cohort of 461 CKCS including 187 unaffected and 274 SM-affected (that included the original 65 dogs) were genotyped with two TagSNPs from each of the associated regions on CFA22 and CFA26. While the 2 selected TagSNPs on CFA26 at position 32,797,595 bp and $32,757,080$ bp did not show any significant association to $\mathrm{SM}$ ( $P$ value $=0.7637$ and 0.7614$)$, the 2 selected TagSNPs on CFA22 at position $13,933,606$ bp and $13,804,718$ bp reached significance $(P$ value $=0.0104$ and $0.02309)$. Bonferroni corrected $P$ value of the TagSNP at position 13,933,606 bp on CFA22, was still significant at a $\mathrm{P}$ value of 0.0104 . Hence, we successfully identified a region on CFA22 associated to ratio $\mathrm{F}-\mathrm{d} / \mathrm{BC}$ and $\mathrm{SM}$ in the CKCS dogs.

\section{Discussion}

Studies of CM in large affected CKCS pedigrees suggested a polygenic inheritance with a wide clinical spectrum where CM with SM represents the most aggravated form $[11,25]$. The genetic factors predisposing to CM and SM have been shown to be interrelated and seem to have their origin in bone development with hypoplasia of the supra and basisoccipital bones and reduced caudal fossa volume associated with a compensatory increase in height of the cranial fossa [7]. Other associated abnormalities include: reduced occipital crest; rostral displacement of the atlas and axis (atlantooccipital overlapping); medulla oblongata elevation/kinking; more acute angulation of the axis bone to the cranial bases (cervical flexure); more acute angle at the spheno-occipital synchondrosis (sphenoid flexure) $[4,6,7,26,27]$; reduced volume of jugular foramen and venous sinus $[28,29]$; a relatively large cerebellum $[10,30,31]$, and dorsal compression from atlantoaxial bands [32,33]. While some of these traits may be a consequence or insufficiency of the occipital sclerotomes (paraxial mesoderm) which form the skull base and parts of the atlas and axis and associated ligaments [1], we cannot exclude a complex origin where multiple genes lead to a range of phenotypes regrouped as CM with SM.

As previously demonstrated, cranial morphometric measurements can provide significant information to decompose the complex nature of CM and SM $[6,16,17]$. In this study, we identified 6 traits (line $\mathrm{AE}$, line $\mathrm{AI}$, angle 3 , angle 7 , ratio $\mathrm{F}-\mathrm{d} / \mathrm{BC}$ and $L 4+L 7$ ) significantly associated to STD size. Genetic investigation of these traits identified significant association with SNPs on CFA15 with F-d/BC and on CFA26 with $L 4+L 7$. Both these traits represented a combination that demonstrated a reduction in the overall size of the caudal cranial fossa and rearrangement of the neural parenchyma (Fig. 2). Screening of SNPs that were suggestive of association identified one SNP on CFA22, BICF2P1045632, associated with $\mathrm{F}-\mathrm{d} / \mathrm{BC}$, that mapped to a region found to be associated with the line $\mathrm{BC}$ in a previous association study of CM in the GB breed [17]. This replication

Table 4 Raw and permutation P values of the F-d/BC-associated haplotypes in the CFA26 candidate region at 32226403$33034398 \mathrm{bp}$

\begin{tabular}{lllll}
\hline Name & Haplotype & Frequency & Raw $P$ value & Corrected $P$ value \\
\hline $\mathbf{1}$ & TTCCCGACAGACGGTAATTGGTTATAATGTA & 0.215 & 0.00237 & 0.01067 \\
$\mathbf{2}$ & CCGTAAGTAAGAGGCAGCGCGTCTCGAACCG & 0.492 & 0.903 & 1 \\
$\mathbf{3}$ & TICCAAGTAAGAAGGCAGCGCGTCTCGAAACCG & 0.0538 & 0.562 & 0.998 \\
$\mathbf{4}$ & TICCCGACAGACGGTAATGTGTTCGAACCG & 0.0308 & 0.434 & 0.9865 \\
$\mathbf{5}$ & CTCTAAGTGAACAATAGGCATACTTCGGAGCTG & 0.108 & 0.000584 & 0.00231 \\
$\mathbf{6}$ & CTGTCAATGAACAGTAGGCATACTTCGGAGCTG & 0.0231 & 0.0584 & 0.3657 \\
$\mathbf{7}$ & CCGTAAGTAAGCAATAGGCATACTTCGAGCTG & 0.0154 & 0.724 & 1 \\
\hline
\end{tabular}




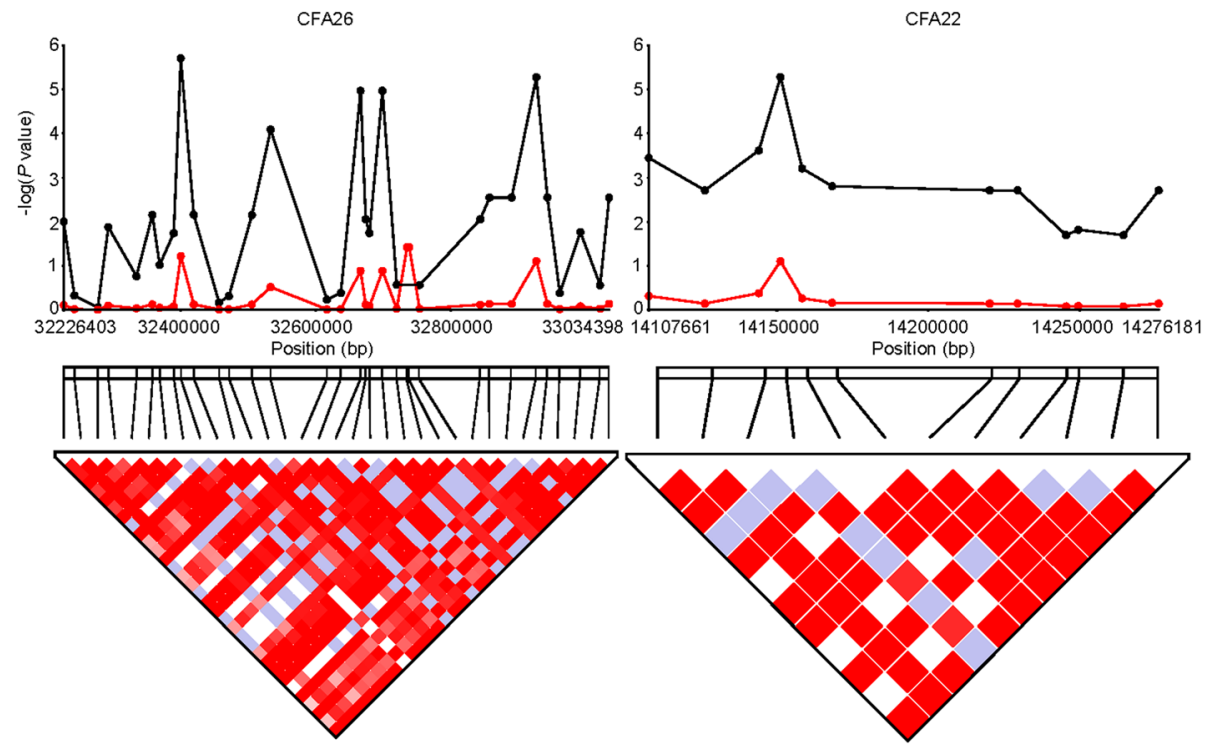

Fig. 5 P value distribution inside the CFA26 (32226403-33034398 bp) and the CFA22 (14107661-14276181 bp) candidate loci. Top graphs represent the $P$ values before correction (black line) and after FDR correction (red line) for the SNPs in reconstructed regions

strengthens the candidacy of the locus on CFA22 for further genetic investigation. Haplotype analysis of candidate loci on CFA15, CFA26 and CFA22 identified significantly associated haplotypes only on CFA22 and CFA26 loci, but none on the CFA15 locus. This suggests that CFA15 initial scores were driven by a rare haplotype overrepresented in extreme cases. Line $\mathrm{BC}$ measures the distance from the caudal end of the basiocciput to the atlas, across the foramen magnum. Previous association of line $\mathrm{BC}$ to $\mathrm{CM}$ in $\mathrm{GB}$ strengthens the candidacy of $\mathrm{F}-\mathrm{d} / \mathrm{BC}$ as an important factor in $\mathrm{CM}$ and $\mathrm{SM}$ etiology $[16,17] . L 4+L 7$ was found to be smaller in SM dogs as compared to no SM dogs in linear regression analyses of SM-significantly associated traits. This suggests that the reduced caudal cranial fossa representing CM has a direct influence on SM. Replication of these findings in a larger cohort could provide better diagnostic tools of $\mathrm{SM}$ in CM-affected dogs.

Genotyping of tagSNPs selected from both candidate loci on CFA22 and CFA26 in an extended cohort of 461 CKCS (187 SM unaffected, 274 SM-affected) identified a significant association of the candidate locus on CFA22 and SM. Hence, this candidate locus that was suggestive of association to the ratio $\mathrm{F}-\mathrm{d} / \mathrm{BC}$ in CKCS and to the line $\mathrm{BC}$ in a previous association study in $\mathrm{GB}$ dogs was found to be significantly associated to SM, strengthening its candidacy for SM. On the other hand, the tagSNPs on CFA26 that was significantly associated to $L 4+L 7$ were not significantly associated to SM in the larger cohort. In a parallel study which characterized the phenotype of $\mathrm{CM}$ in the CKCS, two different skull conformation anomalies were identified which resulted in SM in this breed [6].
The trait of smaller $L 4+L 7$ was a feature of one conformation anomaly (case 2, Fig. 5 in reference [6]). It is therefore possible that significance of this trait in the larger CKCS cohort was reduced since other associated features were underrepresented. By contrast, higher F-d/BC increased risk of SM in both CKCS skull anomalies.

The associated loci on CFA22 and CFA26 harboured each only one gene: $P C D H 17$ and ZWINT (respectively). Targeted next generation sequencing of both CFA22 and CFA26 candidate loci identified a total of 37 and 339 significantly associated SNPs with ratio F-d/BC and $L 4+L 7$ respectively. No mutation in the coding region of either gene was detected, except for one synonymous mutation in PCDH17. We hypothesize that predisposing mutations in these two regions are most likely regulatory that would affect RNA expression of either PCDH17 or ZWINT or other unannotated transcripts. Alternatively, these regulatory mutations could have a long-range expression effect on transcripts residing outside the 2 candidate regions. RNA-sequencing or quantitative RT-PCR studies in affected tissues from dogs carrying the associated haplotypes are needed to test this hypothesis.

PCDH17 (PROTOCADHERIN 17) belongs to the family of protocadherins that are involved in the adhesion and sorting of cells during tissue morphogenesis. It is expressed specifically in several regions of the developing and adult brain and spinal cord [34-41]. It regulates spine development, presynaptic assembly, vesicle accumulation and transmission in corticobasal ganglia synapses [34]. Overexpression of $P C D H 17$ in primary cortical neurons is associated with significantly decreased dendritic spine density and abnormal dendritic morphology 
[42] and it is possible that variants in the dog gene could play a role in development of the neural tissue and affect $\mathrm{CM} / \mathrm{SM}$ disease expressivity. Additional knockout studies in cell or animal models are needed to further investigate the potential role of this gene in the pathogenesis of SM. ZWINT (ZW10-INTERACTOR) has an important role in kinetochore assembly and proper chromosome segregation [43]. In rats, it was shown to be expressed in different regions of the brain and in dorsal horn laminae and its expression levels increased with the onset of neuropathic pain after chronic constriction injury of the sciatic nerve [44-46]. We tested for association between the SM-associated locus on CFA26 and pain in our cohort of 65 CKCS and we did not detect any significant association. However, at the time of DNA collection, phenotyping for pain was not as rigorous and pain relating to CM versus SM pain was not separated. Moreover, objective phenotyping for pain, a subjective experience, is extremely difficult especially when it is partly dependent on owner reporting. Genetic investigation in larger CKCS cohorts with improved pain phenotyping data is needed to further analyze the role of ZWINT in pain development and in SM associated with CM.

$\mathrm{CM}$ with $\mathrm{SM}$ in the dog is very similar to a condition in humans called Chiari malformation I (CMI) with a reported frequency of 1 in 1280. As in dogs, the prevalence of SM secondary to CMI in humans is high reaching $65 \%-85 \%[47,48]$. Genetic studies of CM and $\mathrm{SM}$ in both humans and dogs clearly suggest a complex genetic architecture which has hampered the identification of predisposing genetic factors. The dog model is the only known naturally-occurring animal model for CMI in humans. The reduced genetic variability of dogs caused by founder effects, genetic bottlenecks and strong inbreeding make it an excellent tool for investigation of complex diseases [49]. Hence, gene identification studies in CM with SM in the dog might provide an entry point for identification of novel genes and pathways involved in the pathogenesis of CMI and SM in humans.

\section{Conclusions}

In this study, we have used a genome-wide association study to decipher the genetics of SM secondary to CM in the CKCS breed. We identified 6 cranial T1-weighed sagittal MRI measurements that were associated with the syrinx transverse diameter. We next identified 2 haplotypes on CFA22 and CFA26 that were significantly associated to ratio F-d/BC and $L 4+L 7$ respectively. Genotyping of a larger cohort of CKCS dogs confirmed association of the locus on CFA22 with SM in this breed. Each of these 2 haplotypes harbored only one gene: $P C D H 17$ on CFA22 that codes for a cell adhesion molecule specifically expressed in the brain and spinal cord and ZWINT that plays a role in proper chromosome segregation and whose expression is increased with the onset of neuropathic pain. Additional molecular genetic studies in larger CKCS cohorts from various affected brachycephalic breeds and in cell and animal models are needed to further investigate the role of the 2 associated loci and the genes they harbor in the pathogenesis of SM secondary to CM. Our study represents an essential step towards a better understanding of the complex genetics of this devastating condition and development of breeding strategies that aim at eliminating it from the affected dog breeds. It also provides an important model for studying CMI/SM in humans.

\section{Additional files}

Additional file 1: Table S1. Characteristics and measurements of 96 CKCS of the cohort. This table includes the gender, age, clinical status and all MRI cranial measurements taken on the 96 CKCS dogs included in this study. (XLSX $41 \mathrm{~kb})$

Additional file 2: Table S2. SNPS suggestive of association to SM in the CKCS breed. This table enlists all SNPS suggestive of association with FDR corrected scores between 0.05 and 0.1. These SNPs were identified following a GWAS using a mixed linear model with age as a covariate on the previously identified traits (Line $A E$, line $A l$, angle 3, angle 7, ratio F-d/ $B C$ and $L 4+L 7)$. (DOCX $15 \mathrm{~kb}$ )

Additional file 3: Figure S1. $P$ value distribution inside the CFA15 (24537882-26,252,411 bp) associated region. This region spans 1.7 Mb surrounding SNPs associated to ratio F-d/BC and was identified using Haploview V4.2. (PDF 54 kb)

\section{Abbreviations}

CFA: Canis familiaris autosome; CM: Chiari-like malformation;

CSF: Cerebrospinal fluid; DICOM: Digital Imaging and Communications in Medicine; GB: Griffon Bruxellois; GWAS: Genome-wide association study; Mb: Mega basepairs; MRI: Magnetic Resonance Imaging; PCDH17: Protocadherin 17; SM: Syringomyelia; STD: Syrinx transverse diameter; TagSNPs: Tagging single nucleotide polymorphisms; ZWINT: ZW10interactor

\section{Acknowledgements}

Thanks are given to the dedication and generosity of the many Cavalier King Charles owners worldwide who participated in, or supported the research into CM/SM. In particular, Dana Schuller-Kuyper, Sandy Smith, Margaret Carter and members of the Companion Cavalier King Charles Club, Maggie Ford and members of the Cavalier Club UK, Karlin Lillington, Nickie Hughes and the Cavalier Talk Forum, Pat Barrington and the Cavalier Fanciers of Southern Ontario and the staff at Fitzpatrick Referrals and Stone Lion Veterinary Hospital.

Availability of data and materials Raw and processed data for the 65 dogs with genotypes are available at http://www.ncbi.nlm.nih.gov/geo/query/acc.cgi?acc=GSE102906

\section{Funding}

This study was mainly supported by funds from the Dog Trust foundation https://www.dogstrust.org.uk/ (Z.K., C.R., S.P.K. and F.A.). MRI costs were partially covered by 'Syringomyelia DNA Research Fund (http:// www.veterinary-neurologist.co.uk/Syringomyelia/DNA_Research/), Cavalier Matters Charity (http://www.cavaliermatters.org/) and 'For the Love of Ollie' (http://www.cavacare.org/). P.L. is awarded by the CHU Sainte Justine Foundation (www.fondation-sainte-justine.org/) and the Fonds de Recherche du Québec-Santé (www.frqs.gouv.qc.ca/). S.P.K. doctorate studies are supported by Cavalier Matters Charity. 


\section{Authors' contributions}

FA and PL contributed to the study design, analyzed the data from the genome-wide association study, targeted next generation sequencing and tagSNP association study and were major contributors in writing the manuscript. SPK contributed to the study design, was a major contributor in cohort recruitment and selection, analyzed all DICOM T1-weighted midsagittal MRI images and contributed to writing the manuscript. KK, SG, GBC, JS, PM contributed to cohort recruitment and MRI data acquisition. GR contributed to preparing DNA samples. CR was a major contributor to the study conception and design and data acquisition, contributed to revising the manuscript. ZK was a major contributor to study conception and design, data interpretation and writing the manuscript. All authors read and approved the final manuscript.

\section{Ethics approval}

MRI of the brain and cervical region were obtained either for diagnostic reasons or for screening prior to breeding. Blood or saliva was withdrawn at the end of the MRI procedure whilst the dog is still under the effect of the anaesthesia ensuring minimal stress to the animal. In the United Kingdom (UK), Home Office regulations restrict blood sampling for non-diagnostic reasons however if the dog had a blood sample taken for a veterinary diagnostic test and a small amount of excess blood in EDTA remained then this sample was submitted to the study. For majority of UK owned dogs, the DNA was collected non-invasively via a sponge that absorbs saliva in the mouth (Oragene-ANIMAL, DNA Genotek, Inc). It was not necessary to obtain approval from an ethical committee as the procedures performed were the necessary diagnostic tests for the animals undergoing veterinary treatment. Written consent was obtained from all owners and actual identity of dogs remained anonymous. It is important to note that dogs included in this study were not experimental animals but animals undergoing appropriate veterinary treatment or British Veterinary Association/Kennel Club health screening for breeding purposes and therefore no additional welfare considerations were required.

\section{Consent for publication}

Not applicable.

\section{Competing interests}

The authors declare that they have no competing interests.

\section{Publisher's Note}

Springer Nature remains neutral with regard to jurisdictional claims in published maps and institutional affiliations.

\section{Author details}

'Department of Neurosciences, CHU Sainte Justine Research Center, University of Montréal, 3175 Cote-Sainte-Catherine, Room 3.17.006, Montreal, QC H3T 1C5, Canada. ${ }^{2}$ School of Veterinary Medicine, Faculty of Health \& Medical Sciences, University of Surrey, Guildford, Surrey GU2 7AL, UK. ${ }^{3}$ Department of Medical Imaging, London Health Sciences Centre, London, ON N6A 5A5, Canada. ${ }^{4}$ Stone Lion Veterinary Hospital, 42 High Street, Wimbledon SW19 5AU, UK. ${ }^{5}$ Dick White Referrals, Six Mile Bottom, Cambridgeshire CB8 OUH, UK. ${ }^{6}$ Thames Valley Veterinary Services, London, ON N6A 4V2, Canada. ${ }^{7}$ Department of Clinical Sciences of Companion Animals, Faculty of Veterinary Medicine, Utrecht University, Utrecht 3584, CM, The Netherlands. ${ }^{8}$ Montreal Neurological Institute and McGill University, Montreal, QC H3A 2B4, Canada. ${ }^{9}$ Fitzpatrick Referrals, Godalming, Surrey GU7 2QQ, UK.

Received: 27 July 2017 Accepted: 15 March 2018

Published online: 22 March 2018

\section{References}

1. Flint G, Rusbridge C. Syringomyelia: A Disorder of CSF Circulation. 2nd ed. Heidelberg: Springer Berlin; 2016.

2. Parker JE, Knowler SP, Rusbridge C, Noorman E, Jeffery ND. Prevalence of asymptomatic syringomyelia in Cavalier King Charles spaniels. Vet Rec. 2011; 168:667.

3. Nalborczyk ZR, McFadyen AK, Jovanovik J, Tauro A, Driver CJ, Fitzpatrick N, et al. MRI characteristics for "phantom" scratching in canine syringomyelia. BMC Vet Res. 2017;13:340.
4. Cerda-Gonzalez S, Olby NJ, Broadstone R, McCullough S, Osborne JA. Characteristics of cerebrospinal fluid flow in Cavalier King Charles Spaniels analyzed using phase velocity cine magnetic resonance imaging. Vet Radiol Ultrasound. 2009:50:467-76.

5. Driver CJ, De Risio L, Hamilton S, Rusbridge C, Dennis R, McGonnell IM, et al. Changes over time in craniocerebral morphology and syringomyelia in cavalier King Charles spaniels with Chiari-like malformation. BMC Vet Res. 2012;8:215.

6. Knowler SP, Cross C, Griffiths S, McFadyen AK, Jovanovik J, Tauro A, et al. Use of Morphometric Mapping to Characterise Symptomatic Chiari-Like Malformation, Secondary Syringomyelia and Associated Brachycephaly in the Cavalier King Charles Spaniel. PLoS One. 2017;12:e0170315.

7. Knowler SP, Kiviranta A-M, McFadyen AK, Jokinen TS, La Ragione RM, Rusbridge C. Craniometric Analysis of the Hindbrain and Craniocervical Junction of Chihuahua, Affenpinscher and Cavalier King Charles Spaniel Dogs With and Without Syringomyelia Secondary to Chiari-Like Malformation. PLoS One. 2017;12:e0169898.

8. Mitchell TJ, Knowler SP, van den Berg H, Sykes J, Rusbridge C. Syringomyelia: determining risk and protective factors in the conformation of the Cavalier King Charles Spaniel dog. Canine Genet Epidemiol. 2014;1:9.

9. Schmidt MJ, Neumann AC, Amort KH, Failing K, Kramer M. Cephalometric measurements and determination of general skull type of Cavalier King Charles Spaniels. Vet Radiol Ultrasound. 2011;52:436-40.

10. Shaw TA, McGonnell IM, Driver CJ, Rusbridge C, Volk HA. Increase in cerebellar volume in Cavalier King Charles Spaniels with Chiari-like malformation and its role in the development of syringomyelia. PLoS One. 2012;7:e33660.

11. Lewis T, Rusbridge C, Knowler P, Blott S, Woolliams JA. Heritability of syringomyelia in Cavalier King Charles spaniels. Vet J Lond Engl. 2010;183: 345-7.

12. Rusbridge C, Knowler SP. Hereditary aspects of occipital bone hypoplasia and syringomyelia (Chiari type I malformation) in cavalier King Charles spaniels. Vet Rec. 2003;153:107-12.

13. Mateescu RG, Burton-Wurster NI, Tsai K, Phavaphutanon J, Zhang Z, Murphy $\mathrm{KE}$, et al. Identification of quantitative trait loci for osteoarthritis of hip joints in dogs. Am J Vet Res. 2008;69:1294-300.

14. Pfahler S, Distl O. Identification of quantitative trait loci (QTL) for canine hip dysplasia and canine elbow dysplasia in Bernese mountain dogs. PLoS One. 2012;7:e49782.

15. Schoenebeck JJ, Hutchinson SA, Byers A, Beale HC, Carrington B, Faden DL, et al. Variation of BMP3 contributes to dog breed skull diversity. PLoS Genet. 2012;8:e1002849.

16. Knowler SP, McFadyen AK, Freeman C, Kent M, Platt SR, Kibar Z, et al. Quantitative analysis of Chiari-like malformation and syringomyelia in the Griffon Bruxellois dog. PLoS One. 2014;9:e88120.

17. Lemay $P$, Knowler SP, Bouasker $S$, Nédélec $Y$, Platt $S$, Freeman $C$, et al. Quantitative trait loci (QTL) study identifies novel genomic regions associated to Chiari-like malformation in Griffon Bruxellois dogs. PLoS One. 2014:9:e89816.

18. Knowler SP, v/d Berg H, McFadyen A, La Ragione RM, Rusbridge C. Inheritance of Chiari-Like Malformation: Can a Mixed Breeding Reduce the Risk of Syringomyelia? PLoS One. 2016;11:e0151280.

19. Rusbridge C, Carruthers H, Dubé M-P, Holmes M, Jeffery ND. Syringomyelia in cavalier King Charles spaniels: the relationship between syrinx dimensions and pain. J Small Anim Pract. 2007:48:432-6.

20. R Core Team. R: A language and environment for statistical computing. Vienna: R Foundation for StatisticalComputing; 2013. http://www.R-project.org/.

21. Zhou X, Stephens M. Efficient multivariate linear mixed model algorithms for genome-wide association studies. Nat Methods. 2014;11:407-9.

22. AJ Bass, Dabney A and Robinson D. qvalue: Q-value estimation for false discovery rate control. R package version 2.8.0, 2015 http://github.com/ jdstorey/qualue.

23. Barrett JC, Fry B, Maller J, Daly MJ. Haploview: analysis and visualization of LD and haplotype maps. Bioinform Oxf Engl. 2005;21:263-5.

24. Purcell S, Neale B, Todd-Brown K, Thomas L, Ferreira MAR, Bender D, et al. PLINK: a tool set for whole-genome association and population-based linkage analyses. Am J Hum Genet. 2007;81:559-75.

25. Rusbridge C, Knowler P, Rouleau GA, Minassian BA, Rothuizen J. Inherited occipital hypoplasia/syringomyelia in the cavalier King Charles spaniel: experiences in setting up a worldwide DNA collection. J Hered. 2005: 96:745-9. 
26. Cerda-Gonzalez S, Olby NJ, Griffith EH. Medullary position at the craniocervical junction in mature cavalier King Charles spaniels: relationship with neurologic signs and syringomyelia. J Vet Intern Med. 2015;29:882-6.

27. Marino DJ, Loughin CA, Dewey CW, Marino LJ, Sackman JJ, Lesser ML, et al. Morphometric features of the craniocervical junction region in dogs with suspected Chiari-like malformation determined by combined use of magnetic resonance imaging and computed tomography. Am J Vet Res. 2012;73:105-11.

28. Fenn J, Schmidt MJ, Simpson H, Driver CJ, Volk HA. Venous sinus volume in the caudal cranial fossa in Cavalier King Charles spaniels with syringomyelia. Vet J Lond Engl. 2013;197:896-7.

29. Schmidt MJ, Ondreka N, Sauerbrey M, Volk HA, Rummel C, Kramer M. Volume reduction of the jugular foramina in Cavalier King Charles Spaniels with syringomyelia. BMC Vet Res. 2012;8:158.

30. Cross HR, Cappello R, Rusbridge C. Comparison of cerebral cranium volumes between cavalier King Charles spaniels with Chiari-like malformation, small breed dogs and Labradors. J Small Anim Pract. 2009;50:399-405.

31. Driver CJ, Rusbridge C, Cross HR, McGonnell I, Volk HA. Relationship of brain parenchyma within the caudal cranial fossa and ventricle size to syringomyelia in cavalier King Charles spaniels. J Small Anim Pract. 2010;51:382-6.

32. Cerda-Gonzalez S, Olby NJ, Griffith EH. Dorsal compressive atlantoaxial bands and the craniocervical junction syndrome: association with clinical signs and syringomyelia in mature cavalier King Charles spaniels. J Vet Intern Med. 2015;29:887-92.

33. Cerda-Gonzalez S, Olby NJ, McCullough S, Pease AP, Broadstone R, Osborne JA. Morphology of the caudal fossa in Cavalier King Charles Spaniels. Vet Radiol Ultrasound. 2009;50:37-46.

34. Hoshina N, Tanimura A, Yamasaki M, Inoue T, Fukabori R, Kuroda T, et al. Protocadherin 17 regulates presynaptic assembly in topographic corticobasal Ganglia circuits. Neuron. 2013;78:839-54.

35. Lin J, Wang C, Redies C. Expression of delta-protocadherins in the spinal cord of the chicken embryo. J Comp Neurol. 2012;520:1509-31.

36. Liu Q, Chen Y, Pan JJ, Murakami T. Expression of protocadherin-9 and protocadherin-17 in the nervous system of the embryonic zebrafish. Gene Expr Patterns GEP. 2009;9:490-6.

37. Liu Q, Bhattarai S, Wang N, Sochacka-Marlowe A. Differential expression of protocadherin-19, protocadherin-17, and cadherin-6 in adult zebrafish brain. J Comp Neurol. 2015;523:1419-42.

38. Hertel N, Redies C, Medina L. Cadherin expression delineates the divisions of the postnatal and adult mouse amygdala. J Comp Neurol. 2012;520:3982-4012.

39. Krishna-K K, Hertel N, Redies C. Cadherin expression in the somatosensory cortex: evidence for a combinatorial molecular code at the single-cell level. Neuroscience. 2011;175:37-48.

40. Kim S-Y, Chung HS, Sun W, Kim H. Spatiotemporal expression pattern of non-clustered protocadherin family members in the developing rat brain. Neuroscience. 2007;147:996-1021

41. Kim SY, Mo JW, Han S, Choi SY, Han SB, Moon BH, et al. The expression of non-clustered protocadherins in adult rat hippocampal formation and the connecting brain regions. Neuroscience. 2010;170:189-99.

42. Chang H, Hoshina N, Zhang C, Ma Y, Cao H, Wang Y, et al. The protocadherin 17 gene affects cognition, personality, amygdala structure and function, synapse development and risk of major mood disorders. Mol Psychiatry. 2017; https://doi.org/10.1038/mp.2016.231. [Epub ahead of print]

43. Varma D, Salmon ED. The KMN protein network-chief conductors of the kinetochore orchestra. J Cell Sci. 2012;125:5927-36.

44. Peng G, Han M, Du Y, Lin A, Yu L, Zhang Y, et al. SIP30 is regulated by ERK in peripheral nerve injury-induced neuropathic pain. J Biol Chem. 2009;284: 30138-47.

45. Zhang Y-Q, Guo N, Peng G, Wang X, Han M, Raincrow J, et al. Role of SIP30 in the development and maintenance of peripheral nerve injury-induced neuropathic pain. Pain. 2009;146:130-40.

46. Han M, Xiao X, Yang Y, Huang R-Y, Cao H, Zhao Z-Q, et al. SIP30 is required for neuropathic pain-evoked aversion in rats. J Neurosci. 2014;34:346-55.

47. Hurlbert RJ, Fehlings MG. The Chiari malformations. In: Engler G, editor. Spinal Cord Diseases: Diagnosis and Treatment. New York: Marcel Dekker Inc.; 1998. p. 65-100

48. Speer MC, Enterline DS, Mehltretter L, Hammock P, Joseph J, Dickerson M, et al. Review Article: Chiari Type I Malformation with or Without Syringomyelia: Prevalence and Genetics. J Genet Couns. 2003;12:297-311.

49. Karlsson EK, Lindblad-Toh K. Leader of the pack: gene mapping in dogs and other model organisms. Nat Rev Genet. 2008;9:713-25.

\section{Submit your next manuscript to BioMed Central and we will help you at every step:}

- We accept pre-submission inquiries

- Our selector tool helps you to find the most relevant journal

- We provide round the clock customer support

- Convenient online submission

- Thorough peer review

- Inclusion in PubMed and all major indexing services

- Maximum visibility for your research

Submit your manuscript at www.biomedcentral.com/submit 